\title{
Implication of necrosis-linked p53 aggregation in acquired apoptotic resistance to 5-FU in MCF-7 multicellular tumour spheroids
}

\author{
SU YEON LEE, EUI-KYONG JEONG, HYUN MIN JEON, CHO HEE KIM and HO SUNG KANG
}

\author{
Department of Molecular Biology, College of Natural Sciences, Pusan National University, Pusan 609-735, Korea
}

Received February 11, 2010; Accepted March 8, 2010

DOI: 10.3892/or_00000830

\begin{abstract}
Three-dimensional (3D) multicellular tumour spheroids (MTS) have been used as an in vitro model of solid tumours for drug resistance studies because they mimic the growth characteristics of in vivo tumours more closely than in vitro two-dimensional (2D) culture of cancer cell lines. As observed in solid tumours, MTS exhibits a proliferation gradient with outer regions consisting of proliferating cells that surround inner quiescent cells. The innermost cells in core regions undergo cell death mostly by necrosis to form necrotic core due to insufficient supply of oxygen and nutrient such as glucose with increasing size of spheroids. Tumour necrosis is thought to indicate a poor prognosis and to contribute to acquisition of chemoresistance in solid tumours; however, the mechanism underlying necrosis-mediated chemoresistance remains unclear. In this study, we examined the chemoresistance to 5-Fluorouracil (5-FU) using MCF-7 breast cancer MTS. 5-FU $(400 \mu \mathrm{M})$ induced apoptosis in MCF-7 cell monolayer as determined by HO/PI staining, PARP cleavage, p53 induction, Bax induction, and Bcl-2 downregulation. When MCF-7 breast tumour spheroids were cultured on agarose for 8 days, they reached $\sim 700 \mu \mathrm{m}$ in diameter, with a necrotic core. We found that 5-FU-induced apoptosis is markedly reduced in spheroids that were cultured for 9 days and had necrotic core, compared with MCF-7 monolayer cells and spheroids that were cultured for 6 days and had no necrotic core, indicating that the formation of necrotic core may be linked to acquisition of chemoresistance to 5-FU. We also found that a specific set of cellular proteins including p53 was aggregated into a RIPAinsoluble form during MTS culture. Furthermore, most of p53 induced by 5-FU was aggregated in MTS with necrotic core. Our results suggest that necrosis-linked p53 aggregation may contribute to acquired apoptotic resistance to 5-FU in MTS model system.
\end{abstract}

Correspondence to: Dr Ho Sung Kang, Department of Molecular Biology, College of Natural Sciences, Pusan National University, Pusan 609-735, Korea

E-mail: hspkang@pusan.ac.kr

Key words: multicellular tumour spheroids, acquired apoptotic resistance, necrosis, p53 aggregation, 5-Fluorouracil

\section{Introduction}

In vivo human cancer cells often fail to respond to chemotherapeutic agents. The chemoresistance is achieved by various factors including increased drug efflux due to enhanced expression of ATP binding cassette transporter proteins, alterations in drug activation/inactivation or drug targets, or inhibition of apoptotic pathways due to inactivation of the tumour suppressor p53 and/or activation of anti-apoptotic pathways such as PI3K and ERK1/2 (1-4). Most studies on drug resistance were usually conducted using two-dimensional (2D) monolayers. However, the 2D monolayer in vitro model does not exhibit the full range of drug resistance observed in in vivo solid tumours. For this reason, three-dimensional (3D) multicellular tumour spheroids (MTS) have been used as an in vitro model of solid tumours for drug resistance studies because they mimic the growth characteristics of in vivo tumours more closely than cancer cell line monolayers (5-9).

When grown as 3D MTS structures, tumour cells can acquire an additional resistance to apoptosis, referred to as multicellular resistance (MCR). The acquired MCR has been demonstrated to be linked to a limitation of drug diffusion, to the activation of survival pathways such as NF-кB or mTOR, or to the upregulation of p27 (5-9). However, the molecular basis of MCR is not yet clear. MTS closely resembles avascular tumours. As observed in in vivo solid tumours (10-12), MTS exhibits a proliferation gradient with outer regions consisting of proliferating cells that surround inner quiescent cells (13). The innermost cells in core regions undergo cell death mostly by necrosis to form necrotic core due to insufficient supply of oxygen and nutrient such as glucose with increasing size of spheroids, similar with poorly vascularized solid tumours. Necrosis is characterized by the disruption of cellular membranes and the release of cellular components including HMGB1 which promotes cancer cell growth, invasion, and progression (14-18). Tumour necrosis has been shown to indicate a poor prognosis $(19,20)$ and to contribute to acquisition of chemoresistance in solid tumours, including breast cancer, non-small cell lung cancer, and gastroinstinal stromal tumours (21). However, the mechanism underlying necrosis-mediated chemoresistance remains to be clarified.

5-Fluorouracil (5-FU) is widely used in the treatment of breast, colon, and other cancers (22-24). It interferes with nucleoside metabolism and can be incorporated into RNA 
and DNA, causing cytotoxicity and cell death. 5-FU is converted intracellularly to several active metabolites: fluorodeoxyuridine monophosphate (FdUMP), fluorodeoxyuridine triphosphate (FdUTP) and fluorouridine triphosphate (FUTP). FdUMP forms a stable complex with thymidylate synthase (TS), and thus inhibits production of deoxythymidine monophosphate (dTMP) that is essential for DNA replication and repair. Thus, dTMP depletion results in cytotoxicity. FdUTP and FUTP are incorporated into DNA and RNA, causing DNA and RNA damage, respectively. Chemoresistance to 5-FU is obtained by increased TS expression and by dihydropyrimidine dehydrogenase (DPD), the rate-limiting enzyme in 5-FU catabolism to dihydrofluorouracil (DHFU) in the liver. The tumour suppressor p53 plays a critical role in cell cycle arrest and apoptosis in response to DNA damaging agents such as 5 -FU $(25,26)$. However, in many human cancers, p53 is found mutated, and its activity is reduced (27). For instance, colon cancers retain the mutant p53 allele in $\sim 90 \%$ of the patients. p53 inactivation by specific mutations is a critical step in the development of many cancers, and reduces cellular sensitivity to chemotherapeutic drugs. However, certain cancer cells such as breast cancers contain the wild-type form of p53 protein; for instance, breast cancers retain the mutant allele in only $<40 \%$ of the cases (28). However, breast cancer cells also exhibit resistance to 5-FU, indicating that there is an additional mechanism to overcome the wild-type p53 protein in these cancer cells. Some breast cancers have been shown to inactivate the tumour-suppressing activity of p53 by sequestering it in the cytosol, without mutation.

In this study, we examined the chemoresistance to 5-FU using MCF-7 breast cancer cell MTS. We found that 5-FUinduced apoptosis is markedly reduced in spheroids that were cultured for 9 days and had necrotic core, compared with MCF-7 monolayer cells and spheroids that were cultured for 6 days and had no necrotic core. We also found that most of p53 induced by 5-FU was aggregated into a RIPA-insoluble form in MTS with necrotic core. Our results suggest that necrosis-linked p53 aggregation may contribute to acquired apoptotic resistance to 5-FU in MTS model system.

\section{Materials and methods}

Monolayer and multicellular tumour spheroid (MTS) culture, and 5-FU treatment. Human breast adenocarcinoma cells MCF-7, MDA-MB-231, and MDA-MB-361 were obtained from American Type Culture Collection and cultured as twodimensional (2D) monolayers in DMEM (WelGene) supplemented with $10 \%(\mathrm{v} / \mathrm{v})$ heat-inactivated FBS and $1 \%$ (v/v) penicillin-streptomycin in a $37^{\circ} \mathrm{C}$ humidified incubator with $5 \% \mathrm{CO}_{2}(29,30)$. For three-dimensional (3D) MTS culture, 400 cells were seeded per well in 96-well plates previously coated with $1.2 \%$ agarose. Each well contained $200 \mu 1$ of tissue culture medium, and the spheroids were fed every other day by carefully aspirating $100 \mu \mathrm{l}$ of spent medium and replacing with the same quantity of fresh medium. 5-FU was purchased from Sigma. 5-FU was dissolved in dimethyl sulfoxide and then divided into aliquots and stored at $-70^{\circ} \mathrm{C}$ until used. The stocks were freshly diluted in culture medium before experiment.
Hoechst 33258 and propidium iodide (HO/PI) double staining. To determine the cell death mode, HO (Invitrogen) and PI (Invitrogen) double staining was performed $(29,30)$. In 2D culture, cells were seeded at a density of $2.5 \times 10^{5}$ cells $/ \mathrm{ml}$ in 35-mm dishes. After $24 \mathrm{~h}$, the cells were treated with 5-FU for up to $72 \mathrm{~h}$ and stained with $\mathrm{HO}(1 \mu \mathrm{g} / \mathrm{ml})$ and PI $(5 \mu \mathrm{g} / \mathrm{ml})$ for $15 \mathrm{~min}$, and then dissociated with trypsin/EDTA. In 3D culture, equal numbers of spheroids were transferred to $1.2 \%$ agarose-coated $60-\mathrm{mm}$ dishes at each of the days as indicated in the figure legends. The spheroids were treated with 5-FU and trypsinized and then stained with $\mathrm{HO} / \mathrm{PI}$. Additionally, 96-well plates of spheroids were harvested and fixed in $1 \%$ paraformaldehyde. After processing into wax blocks, the spheroids were sectioned and stained with H\&E or HO/PI.

Western blotting. Protein lysates obtained from cells treated with 5-FU were measured using the Bradford assay. Equal amounts of protein lysates were separated by sodium dodecyl sulfate-polyacrylamide gel electrophoresis on $12 \%$ gels, and electrotransferred to NC membrane and analyzed by Western blotting with antibodies to p53, PARP, Bax, Bcl-2, p21, p27, CuZnSOD, MnSOD (Santa Cruz, CA, USA) and tubulin (Biogenex, CA, USA). After incubation with peroxidaseconjugated secondary antibody, protein expression was detected using ECL Western Blotting Detection Reagents (Amersham Biosciences).

RIPA-soluble and RIPA-insoluble fractionation. RIPAsoluble fraction was obtained by lysing cells in RIPA buffer (50 mM Tris $\mathrm{pH} 7.5,200 \mathrm{mM} \mathrm{NaCl}, 1 \%$ Triton X-100, 0.5\% deoxycholate, $0.1 \%$ sodium dodecyl sulfate, $1 \mathrm{mM}$ EDTA) for $20 \mathrm{~min}$ on ice, followed by centrifugation at 14,000 rpm for $15 \mathrm{~min}$ and the RIPA insoluble fraction was suspended in PBS and boiled in 2X Laemmli sample buffer.

Spheroid selective dissociation. MTS were dissociated into subpopulations of cells from different locations in the spheroid as described previously (31). The spheroids were placed in a cylindrical chamber with a 75-m nylon mesh. After washing the spheroids with PBS, they were treated with a dissociation solution containing $0.125 \%$ trypsin (Life Technologies, Inc.) in a phosphate buffer containing $1 \mathrm{mM}$ EDTA and $25 \mathrm{mM}$ HEPES ( $\mathrm{pH} 7.4$ ). Cells dissociated from the spheroids were collected into stirred tubes containing complete medium on ice, while the remaining aggregates remained in the chamber. Cell suspensions were stored on ice until dissociation was complete.

\section{Results and Discussion}

5-FU-induced cell death. We examined the cytotoxic effects of 5-FU in MCF-7 monolayer cell cultures. MCF-7 cells were treated with 5-FU at different concentrations up to $400 \mu \mathrm{M}$. Treatment of 5-FU caused a dose-dependent decrease in the cell viability, with prominent inhibitory effect on cell viability observed at a concentration of $400 \mu \mathrm{M}$. HO/PI nuclear staining was performed to determine the amount and type of cell death. DNA-binding dyes $\mathrm{HO}$ that is known to cross the plasma membrane of all cells, whether they are damaged or not, causing a blue fluorescence of their nuclei and PI that only 


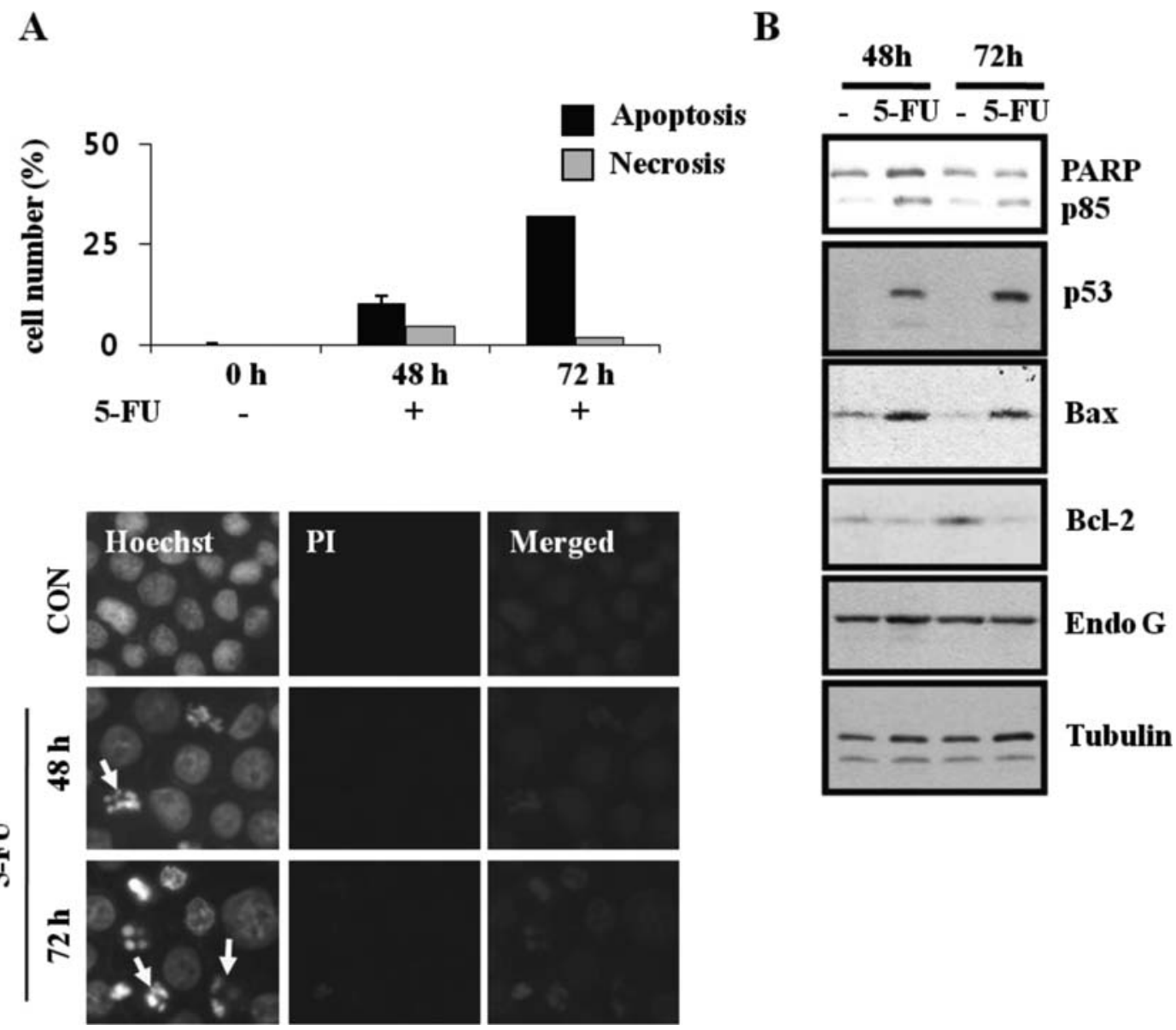

Figure 1. 5-FU induces apoptosis in MCF-7 monolayer cell cultures. (A) MCF-7 cells were treated with $400 \mu \mathrm{M} 5$-FU for 48 and $72 \mathrm{~h}$ and then stained with $\mathrm{HO} / \mathrm{PI}$ and observed with fluorescence microscopy (low panel) and apoptotic and necrotic cells were scored (upper panel). Results (500-800 cells in each group) are expressed as the means \pm SEM from three independent experiments. Arrow indicates apoptotic cells. (B) MCF-7 cells were treated with $400 \mu \mathrm{M}$ 5-FU for 48 and $72 \mathrm{~h}$ and the resulting cell lysates analyzed by Western blotting using antibodies to PARP, p53, Bax, Bcl-2, Endo G, and tubulin.

penetrates cells with damaged membranes and leads to nuclear fluorescence. Thus, intact blue nuclei, condensed/ fragmented blue nuclei, condensed/fragmented pink nuclei, and intact pink nuclei were considered viable, early apoptotic, late apoptotic (secondary necrotic), and necrotic cells, respectively. As shown in Fig. 1A, MCF-7 cells treated with 5-FU at concentrations of $400 \mu \mathrm{M}$ for $72 \mathrm{~h}$ showed the characteristic features of apoptotic cell death, namely nuclear chromatin condensation and the appearance of typical apoptotic bodies. 5-FU is known to induce apoptosis through induction of the tumour suppressor $\mathrm{p} 53$ that induces expression of pro-apoptotic Bax, and suppresses anti-apoptotic Bcl-2. 5-FU $(400 \mu \mathrm{M})$ appeared to induce p53 and Bax and to downregulate Bcl-2 (Fig. 1B). 5-FU also induced cleavage of poly(ADP-ribose) polymerase (PARP), a hallmark of apoptosis.

Formation of MCF-7 multicellular tumour spheroids. To generate spheroid as solid tumour model, we seeded 400 MCF-7 cells to each well of a 96-well plate pre-coated with $1.2 \%$ agarose and the morphology of spheroids in vitro was monitored by light microscopy (Fig. 2A). As MCF-7 cells have an adhesion molecule, E-cadherin, on cell surface, they form tightly packed, rounded spheroids of a homogeneous size that required trypsin treatment and physical strength to disintegrate the spheroids. Multicellular spheroid is known to develop a proliferation gradient, with proliferating cells at the periphery, cell cycle arrested cells in inner regions, and necrotizing cells in core regions (1-3).

To confirm a quiescent state in MTS, the spheroids were selectively dissociated to yield cells from four discrete regions within the spheroid, with each fraction representing $25 \%$ of the total spheroid cell number. We found that increased levels of CKI family proteins, p21waf1/cip1 and p27Kip1 that are primarily involved in the regulation of G1-S progression, were detected in the second and third fractions, but their lower levels were detected in the outermost fraction (first fraction) and in the innermost fraction (fourth fraction) (Fig. 2B). The levels of other proteins such as tubulin were relatively constant throughout all fractions of the spheroids. p53 that is responsible for $\mathrm{p} 21$ waf1/cip1 induction in cell cycle control was also observed in the second fraction (Fig. 2B).

Since p 27 is known to be increased by hypoxia-activated HIF-1 $\alpha$, the second and third fractions are likely to represent a hypoxic region. When MCF-7 breast tumour spheroids were cultured on agarose for 8 days, they reached $\sim 700 \mu \mathrm{m}$ in diameter (Fig. 1A). As observed previously (unpublished data), necrotic core was observed in spheroids when their size reached $>700 \mu \mathrm{m}$ (Fig. 2C). The necrotic core in tumour spheorids was observed using H\&E staining (Fig. 2C) and 
A
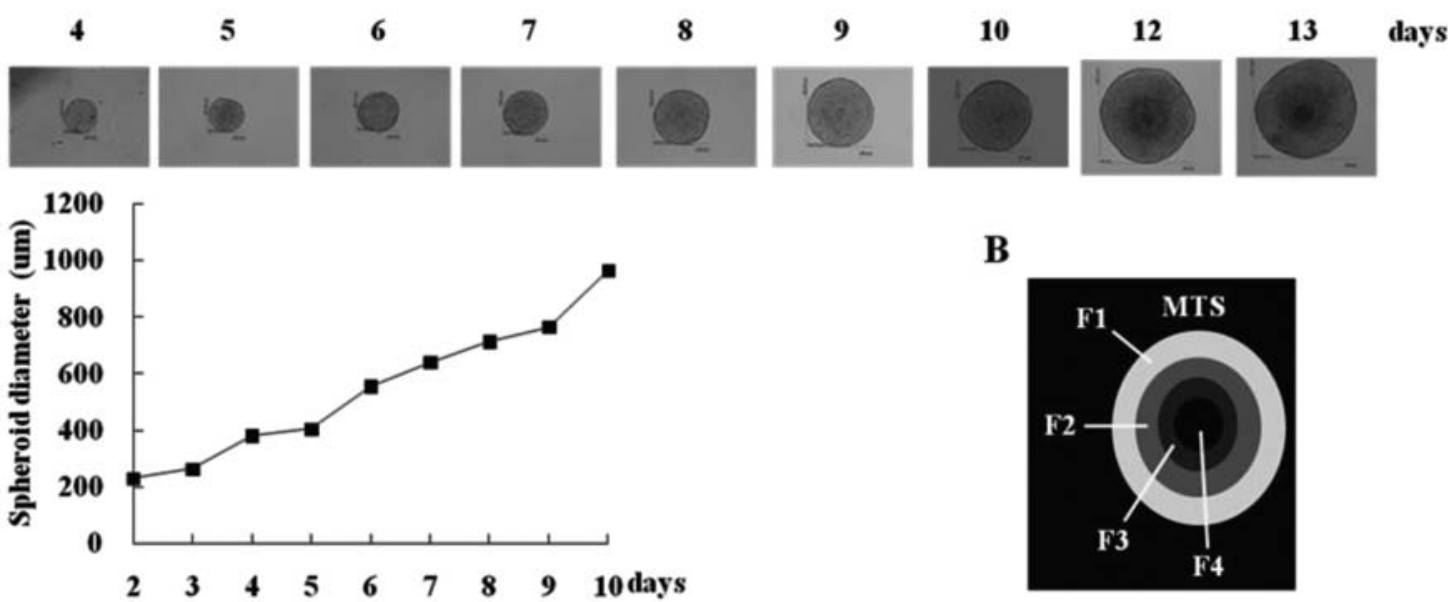

B

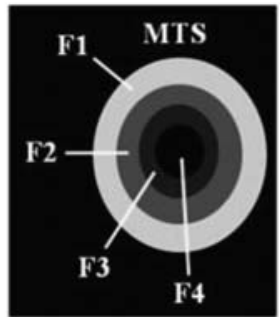

C
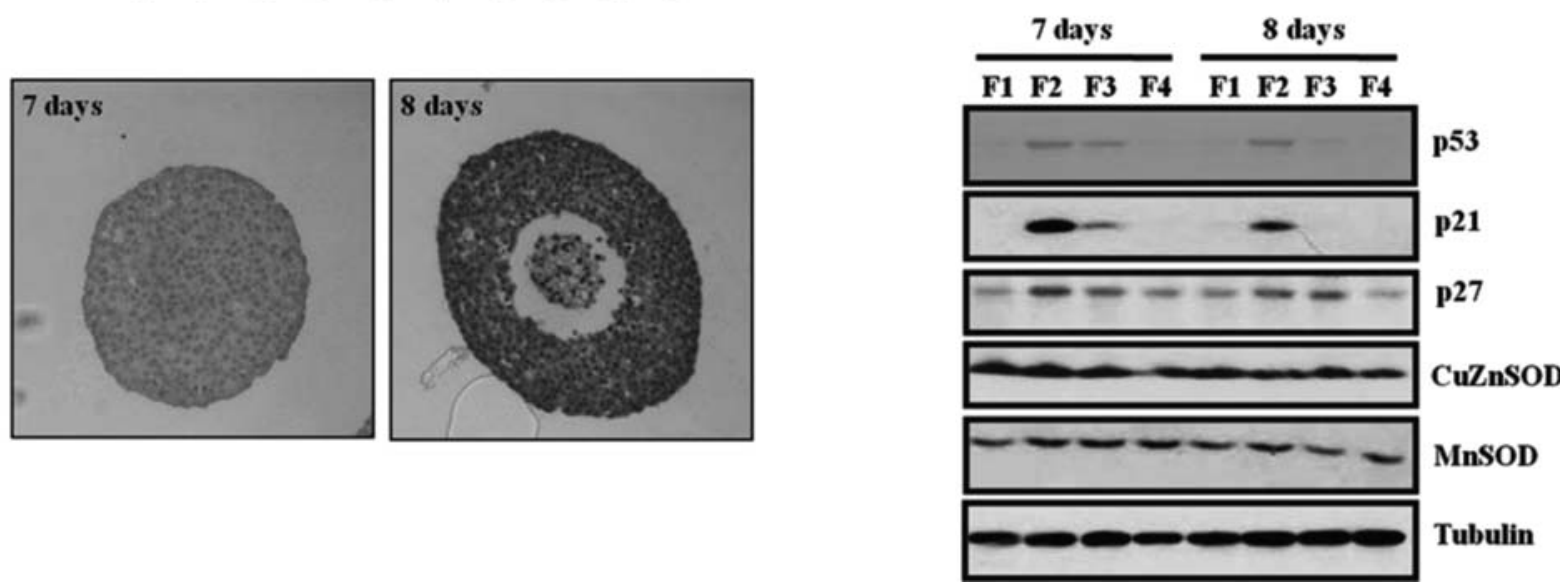

Figure 2. Formation of the necrotic core during MTS culture. (A) A growth curve of MCF-7 cells in MTS culture. MCF-7 cells were seeded into 1.2\% agarose-coated 96-well plates at a density of 400 cells per well and cultured for up to 13 days. To calculate MTS size, diameters of 5 spheroids were measured every day. (B) After 7 and 8 days of MCF-7 MTS culture, MTS were dissociated into subpopulations of cells from different locations in the spheroids as described in Materials and methods. The cells isolated from different locations within MCF-7 spheroids were analyzed by Western blotting using antibodies to p53, p21, p27, CuZnSOD, MnSOD, and tubulin. (C) After 7 and 8 days of MCF-7 MTS culture, MTS were sectioned and stained with H\&E.

HO and PI double staining (data not shown) of paraffin section. The cells in core regions is likely to die by necrosis due to insufficient supply of oxygen and nutrient such as glucose.

Acquisition of chemoresistance in MCF-7 spheroids after emergence of necrotic core. We next examined 5-FU-induced apoptosis using two different MCF-7 MTS model systems: 1) the spheroids that were collected on 4-7 days and did not contain necrotic core and 2) the MTS that were collected on 8-9 days and contained necrotic core. The MTS were treated with $5-\mathrm{FU}$ in agarose-coated $60-\mathrm{mm}$ dishes and the cells dissociated from the spheroids were stained with the HO/PI. As shown in Fig. 3, the cytotoxicity of 5-FU in 6-day spheroid was similar to that in monolayers. The similar cytotoxcity of 5-FU in monolayers and spheroids has been reported in MGH-U1 cells growing as monolayers or spheroids (32). In addition, in Chinese hamster V79 cells, identical amounts of damage were produced in monolayers and spheroids by 5 -FU (33). However, 5-FU-induced apoptosis was markedly reduced in spheroids that were cultured for 9 days and had a necrotic core, compared with that of monolayers and spheroids that were cultured for 6 days and had no necrotic core (Fig. 3).
These results demonstrated that the formation of necrotic core in tumour spheroids may be linked to drug resistance to 5-FU.

A specific set of proteins including p53 was aggregated during MCF-7 MTS culture. In the course of this study, we found that the amount of RIPA-insoluble proteins was increased during MTS culture (Fig. 4). RIPA-soluble protein and RIPAinsoluble protein patterns were quite different, indicating that a specific set of proteins forms RIPA-insoluble aggregates during MTS culture. While cytosolic CuZnSOD and mitochondrial MnSOD were found only in RIPA-soluble fraction, p53 and tubulin was found in both RIPA-soluble and RIPAinsoluble fractions (Fig. 4). The levels of p53 and tubulin in RIPA-insoluble fractions were increased in MTS that were cultured for 8-9 days and had necrotic core, compared with 6-day MTS without necrotic core.

Previously we demonstrated that glucose depletion, one of the stresses that cause metabolic stress in tumours, induces necrosis in cancer cell lines including A549, HepG2, and MDA-MB231 cells in vitro $(29,30)$ and that during metabolic stress-induced necrosis, many cellular proteins including p53 are aggregated in insoluble forms while some cellular proteins including CuZnSOD, HMGB1, and LDH were released (30). 
A
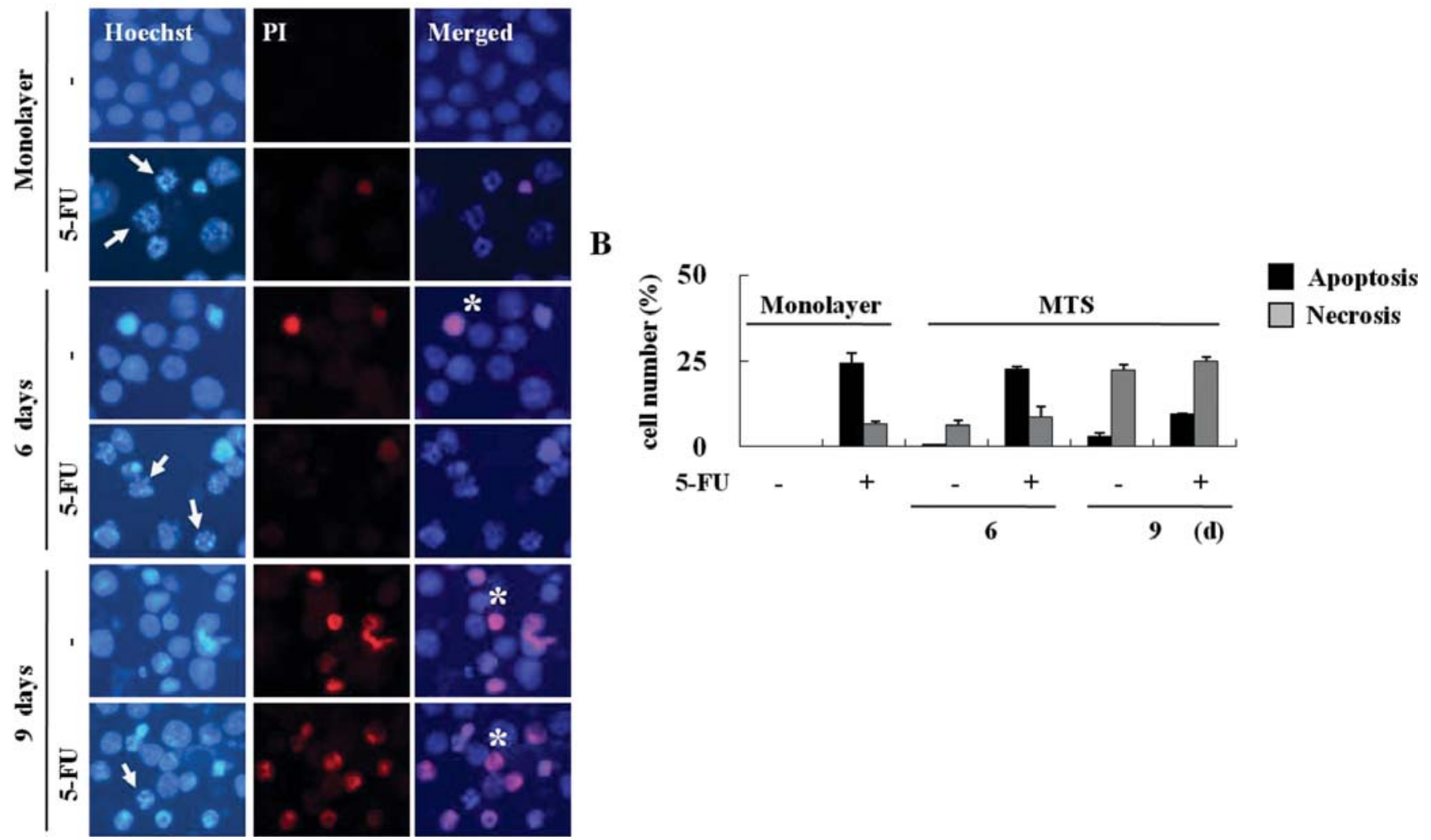

Figure 3. MCF-7 MTS acquire resistance to 5-FU-induced apoptosis. MCF-7 monolayer cells and spheroids cultured for 6 and 9 days were exposed to $400 \mu \mathrm{M}$ 5-FU for additional $72 \mathrm{~h}$. Then the cells were dissociated and stained with HO/PI and observed with fluorescence microscopy (A) and apoptotic and necrotic cells were scored (B). Results (500-800 cells in each group) are expressed as the means \pm SEM from three independent experiments. Arrow indicates apoptotic cells; * indicates necrotizing cells.

A

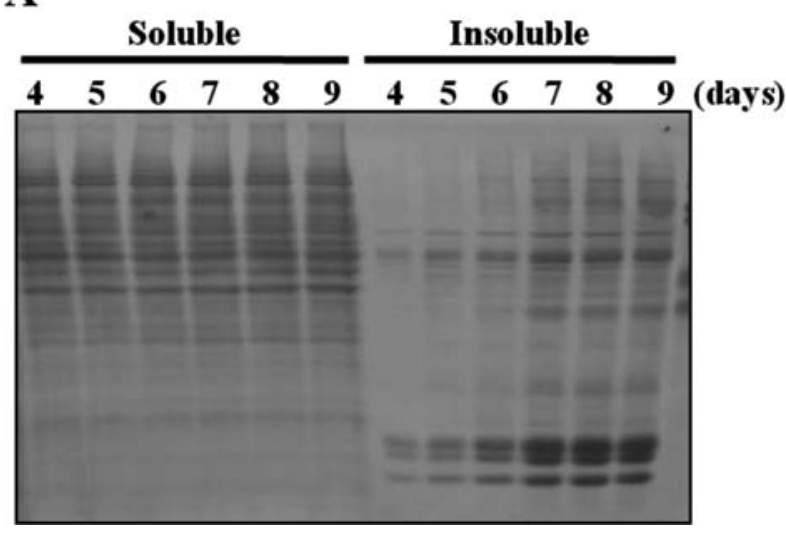

B

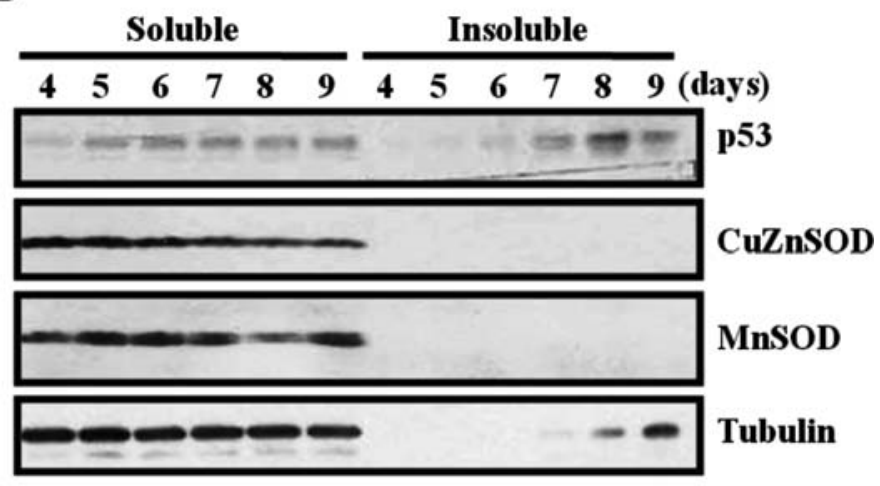

Figure 4. A specific set of proteins including p53 was aggregated during MCF-7 MTS culture. (A and B) MCF-7 cells were seeded into 1.2\% agarose-coated 96-well plates at a density of 400 cells per well and cultured for 4-9 days. MTS were treated with RIPA buffer and RIPA-soluble and RIPA-insoluble fractions were obtained and analyzed by SDS-PAGE and Ponceau S staining (A) and Western blotting with antibodies to p53, CuZnSOD, MnSOD, and tubulin (B).

Intracellular $\mathrm{H}_{2} \mathrm{O}_{2}$ and $\mathrm{O}_{2}^{-}$and mitochondrial ROS play a crucial role(s) in metabolic stress-induced necrosis (29). Since ROS could trigger the formation of oxidatively modified proteins that tend to aggregate, metabolic stress-induced protein aggregation is thought to be mediated by ROS (unpublished data). In MTS system, ROS may be increased in inner regions of MTS as results of metabolic stress due to hypoxia and glucose depletion and may cause protein aggregation and necrosis. In general, necrotic cell death has been suggested to release most cellular proteins due to cell membrane rupture. However, our results show that many cellular proteins form insoluble aggregates, while certain cellular proteins including CuZnSOD, HMGB1, and LDH was selectively released. The molecular mechanism for such 
A

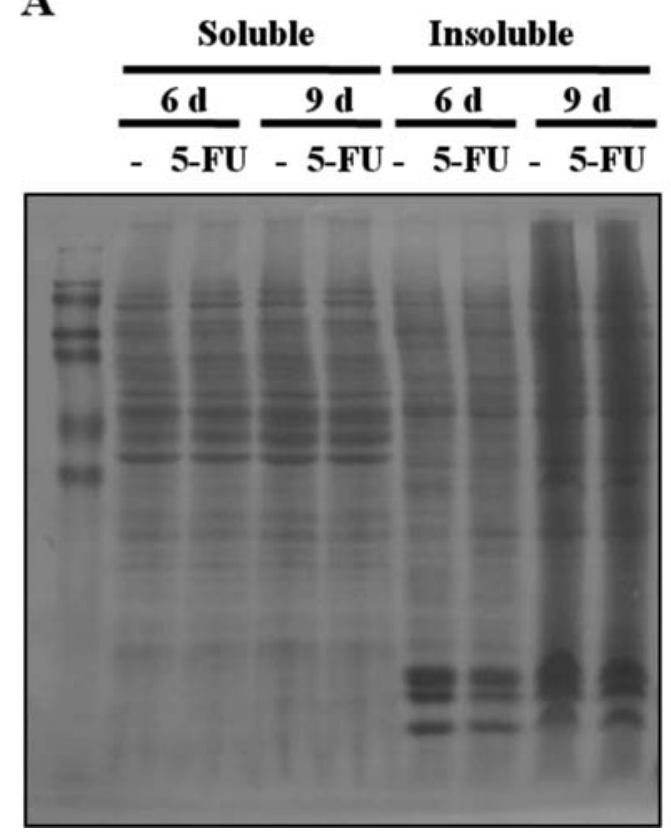

B

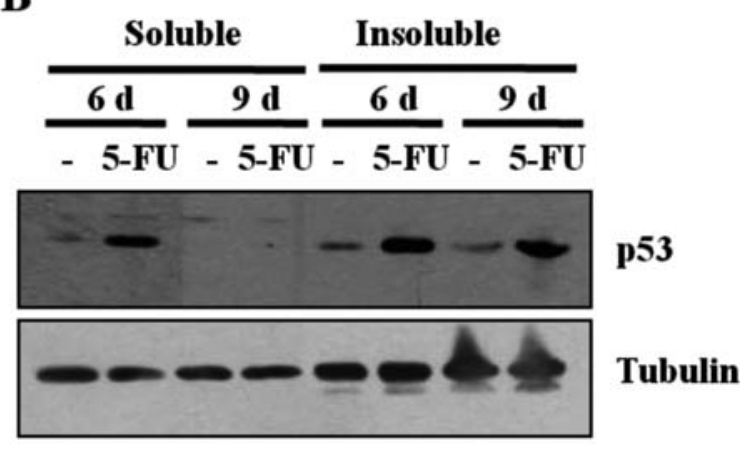

Figure 5. Most of p53 induced by 5-FU was aggregated in MTS with necrotic core. MCF-7 spheroids cultured for 6 and 9 days were exposed to $400 \mu$ M 5-FU for additional $72 \mathrm{~h}$. MTS were then treated with RIPA buffer and RIPA-soluble and RIPA-insoluble fractions were obtained and analyzed by SDS-PAGE and Ponceau S staining (A) and Western blotting with antibodies to p53 (B).

necrosis-linked protein aggregation found in both monolayers and MTS system remains to be elucidated.

Most of p53 induced by 5-FU was aggregated in MTS with necrotic core. When 5-FU was treated to MTS, p53 induction was detected (Fig. 5). However, while p53 induced in 6-day MTS in response to 5-FU was detected in both RIPA-soluble and RIPA-soluble fractions, most of p53 induced in 9-day MTS was detected only in RIPA-insoluble fractions. Thus, p53 induced by $5-\mathrm{FU}$ could be easily aggregated in spheroids with necrotic core. Insoluble p53 could be inactivated in terms of its ability to induce Bax and other target genes. Since 5-FU could induce p53 in MTS at 9 days, the apoptotic resistance to 5-FU at 9 days is not likely to be due to its limited diffusion. 5-FU is known to reach cells at all depths of the spheroid viable rim (32). In addition, examination on sensitivity profiles of cells from various regions within spheroids of Chinese hamster V79 multicell spheroids after treatment with doxorubicin, bleomycin, 5-FU, carmustine, cisplatin, chlorambucil, and mitomycin showed that penetration was a problem only for doxorubicin (34). Our results suggest that necrosislinked p53 aggregation may contribute to acquired apoptotic resistance to 5-FU in MCF-7 MTS.

Biological relevance of this study. p53 negatively regulates cell proliferation and induces apoptosis in response to DNA damage and thus functions as a suppressor of tumourigenesis. In many human cancers, however, p53 is found mutated, and its activity is reduced, and thus, p53 inactivation by specific mutations is the most common mechanism to inhibit the tumour suppressive activities of p53 in the development of many cancers. However, recent studies showed that certain cancer cells such as breast cancers contain the wild-type form of p53 protein (28). Nevertheless, breast cancer cells exhibit resistance to anti-cancer drugs including 5-FU, indicating that there is an additional mechanism to overcome the wildtype p53 protein in these cancer cells. These cancers are characterized by a loss of function of wild-type p53, which accumulates in intracellular aggregates. Actually, accumulation of wild-type inactive p53 has been described in various cancers including neuroblastoma, retinoblastoma, and breast and colon cancers $(28,35-38)$. In these cells, p53 is aggregated in large cytoplasmic and/or nuclear deposits possibly arising from its conformational change. Our results show that in MTS system, ROS may be increased in inner regions of MTS as result of metabolic stress including hypoxia and glucose depletion and may cause protein aggregation including p53.

Necrosis have been shown to contribute to acquisition of chemoresistance in solid tumours (21). Our results suggest that necrosis-linked p53 aggregation may be linked to chemoresistance. In the process of acquiring resistance, the tumour may become cross-resistant to many different types of unrelated drugs. Many anti-cancer drugs have been shown to exert their tumour suppressive activities through inducing and activating $\mathrm{p} 53$. Thus, necrosis-linked $\mathrm{p} 53$ protein aggregation may contribute to tumour cell cross-resistance to other anticancer drugs that exert the tumour preventive activity through p53.

\section{Acknowledgements}

This work was supported by the National Research Foundation of Korea (NRF) grant funded by the Korea government (MEST) (2009-0059210), and a grant of the Korea Healthcare Technology R\&D Project, Ministry for Health, Welfare, Republic of Korea (A080303). 


\section{References}

1. Wilson TR, Longley DB and Johnston PG: Chemoresistance in solid tumours. Ann Oncol 17 (Suppl 10): X315-X324, 2006.

2. Raguz S and Yague E: Resistance to chemotherapy: new treatments and novel insights into an old problem. Br J Cancer 99: 387-391, 2008

3. Vivanco I and Sawyers CL: The phosphatidylinositol 3-Kinase AKT pathway in human cancer. Nat Rev Cancer 2: 489-501, 2002.

4. Balmanno $\mathrm{K}$ and Cook SJ: Tumour cell survival signalling by the ERK1/2 pathway. Cell Death Differ 16: 368-377, 2009.

5. Horning JL, Sahoo SK, Vijayaraghavalu S, Dimitrijevic S, Vasir JK, Jain TK, Panda AK and Labhasetwar V: 3-D tumor model for in vitro evaluation of anticancer drugs. Mol Pharm 5: 849-862, 2008

6. Ivascu A and Kubbies M: Diversity of cell-mediated adhesions in breast cancer spheroids. Int J Oncol 31: 1403-1413, 2007.

7. Barbone D, Yang TM, Morgan JR, Gaudino G and Broaddus VC: Mammalian target of rapamycin contributes to the acquired apoptotic resistance of human mesothelioma multicellular spheroids. J Biol Chem 283: 13021-13030, 2008.

8. Gallardo-Perez JC, Espinosa M, Ceballos-Cancino G, Daniel A, Rodriguez-Enriquez S, Aviles A, Moreno-Sanchez R, Melendez-Zajgla $\mathrm{J}$ and Maldonado V: NF-kappa B is required for the development of tumor spheroids. J Cell Biochem 108: 169-180, 2009.

9. Xing H, Wang S, Hu K, Tao W, Li J, Gao Q, Yang X, Weng D, $\mathrm{Lu} \mathrm{Y}$ and Ma D: Effect of the cyclin-dependent kinases inhibitor p27 on resistance of ovarian cancer multicellular spheroids to anticancer chemotherapy. J Cancer Res Clin Oncol 131: 511-519, 2005 .

10. Gatenby RA and Gillies RJ: Why do cancers have high aerobic glycolysis? Nat Rev Cancer 4: 891-899, 2004.

11. Harris AL: Hypoxia - a key regulatory factor in tumour growth. Nat Rev Cancer 2: 38-47, 2002.

12. Tomes L, Emberley E, Niu Y, Troup S, Pastorek J, Strange K, Harris A and Watson PH: Necrosis and hypoxia in invasive breast carcinoma. Breast Cancer Res Treat 81: 61-69, 2003.

13. Leek RD, Stratford I and Harris AL: The role of hypoxiainducible factor-1 in three-dimensional tumor growth, apoptosis, and regulation by the insulin-signaling pathway. Cancer Res 65 : 4147-4152, 2005.

14. Kanduc D, Mittelman A, Serpico R, Sinigaglia E, Sinha AA, Natale C, Santacroce R, Di Corcia MG, Lucchese A, Dini L, Pani P, Santacroce S, Simone S, Bucci R and Farber E: Cell death: Apoptosis versus necrosis (Review). Int J Oncol 21: $165-170,2002$

15. Zong WX and Thompson CB: Necrotic death as a cell fate. Genes Dev 20: 1-15, 2006.

16. Golstein P and Kroemer G: Cell death by necrosis: towards a molecular definition. Trends Biochem Sci 32: 37-43, 2007.

17. Vakkila $\mathbf{J}$ and Lotze MT: Inflammation and necrosis promote tumour growth. Nat Rev Immunol 4: 641-648, 2004.

18. Lotze MT and Tracey KJ: High-mobility group box 1 protein (HMGB 1): nuclear weapon in the immune arsenal. Nat Rev Immunol 5: 331-342, 2005.

19. Edwards JG, Swinson DE, Jones JL, Muller S, Waller DA and O'Byrne KJ: Tumor necrosis correlates with angiogenesis and is a predictor of poor prognosis in malignant mesothelioma. Chest 124: 1916-1923, 2003

20. Langner C, Hutterer G, Chromecki T, Leibl S, Rehak P and Zigeuner R: Tumor necrosis as prognostic indicator in transitional cell carcinoma of the upper urinary tract. J Urol 176: 910-914, 2006 .
21. Brown JM: Tumor microenvironment and the response to anticancer therapy. Cancer Biol Ther 1: 453-458, 2002.

22. Longley DB, Harkin DP and Johnston PG: 5-fluorouracil: mechanisms of action and clinical strategies. Nat Rev Cancer 3: 330-338, 2003.

23. Zhang N, Yin Y, Xu SJ and Chen WS: 5-Fluorouracil: mechanisms of resistance and reversal strategies. Molecules 13: 1551-1569, 2008.

24. Hernandez-Vargas H, Ballestar E, Carmona-Saez P, von Kobbe C, Banon-Rodriguez I, Esteller M, Moreno-Bueno G and Palacios J: Transcriptional profiling of MCF7 breast cancer cells in response to 5-Fluorouracil: relationship with cell cycle changes and apoptosis, and identification of novel targets of p53. Int J Cancer 119: 1164-1175, 2006.

25. Wyllie A: Apoptosis. Clues in the p53 murder mystery. Nature 389: 237-238, 1997.

26. Tsujimoto Y: Cell death regulation by the Bcl-2 protein family in the mitochondria. J Cell Physiol 195: 158-167, 2003.

27. Bullock AN and Fersht AR: Rescuing the function of mutant p53. Nat Rev Cancer 1: 68-76, 2001.

28. Moll UM, Riou G and Levine AJ: Two distinct mechanisms alter p53 in breast cancer: mutation and nuclear exclusion. Proc Natl Acad Sci USA 89: 7262-7266, 1992.

29. Kim CH, Han SI, Lee SY, Youk HS, Moon JY, Duong HQ, Park MJ, Joo YM, Park HG, Kim YJ, Yoo MA, Lim SC and Kang HS: Protein kinase C-ERK1/2 signal pathway switches glucose depletion-induced necrosis to apoptosis by regulating superoxide dismutases and suppressing reactive oxygen species production in A549 lung cancer cells. J Cell Physiol 211: 371-385, 2007.

30. Han SI, Duong HQ, Choi JE, Lee TB, Kim CH, Lee SY, Jeon HM, Shin SH, Lim SC and Kang HS: Hyperthermia switches glucose depletion-induced necrosis to apoptosis in A549 lung adenocarcinoma cells. Int J Oncol 32: 851-860, 2008.

31. LaRue KE, Khalil M and Freyer JP: Microenvironmental regulation of proliferation in multicellular spheroids is mediated through differential expression of cyclin-dependent kinase inhibitors. Cancer Res 64: 1621-1631, 2004.

32. Erlichman $\mathrm{C}$ and Wu A: Effects of 5-fluorouracil and leucovorin in spheroids: a model for solid tumours. Anticancer Res 11: 671-675, 1991 .

33. Olive PL, Durand RE, Banath JP and Evans HH: Etoposide sensitivity and topoisomerase II activity in Chinese hamster V79 monolayers and small spheroids. Int J Radiat Biol 60: 453-466, 1991.

34. Durand RE: Chemosensitivity testing in V79 spheroids: drug delivery and cellular microenvironment. J Natl Cancer Inst 77: 247-252, 1986.

35. Moll UM, Ostermeyer AG, Haladay R, Winkfield B, Frazier M and Zambetti G: Cytoplasmic sequestration of wild-type p53 protein impairs the G1 checkpoint after DNA damage. Mol Cell Biol 16: 1126-1137, 1996.

36. Ostermeyer AG, Runko E, Winkfield B, Ahn B and Moll UM: Cytoplasmically sequestered wild-type p53 protein in neuroblastoma is relocated to the nucleus by a C-terminal peptide. Proc Natl Acad Sci USA 93: 15190-15194, 1996.

37. Wolff A, Technau A, Ihling C, Technau-Ihling K, Erber R, Bosch FX and Brandner G: Evidence that wild-type p53 in neuroblastoma cells is in a conformation refractory to integration into the transcriptional complex. Oncogene 20: 1307-1317, 2001.

38. Rigacci S, Bucciantini M, Relini A, Pesce A, Gliozzi A, Berti A and Stefani M: The (1-63) region of the p53 transactivation domain aggregates in vitro into cytotoxic amyloid assemblies. Biophys J 94: 3635-3646, 2008. 